\title{
Research on Communication Anti Jamming System based on Multitier Architecture
}

\author{
Jun WANG ${ }^{1, a, *}$, Airong $\mathrm{YU}^{2, \mathrm{~b}}$, Hai SUN ${ }^{3, \mathrm{c}}$, Yong WANG ${ }^{2}$ \\ ${ }^{1}$ Nanjing Telecommunication Institute \\ Nanjing 210007, China \\ ${ }^{2}$ Institute of Command Information Systems \\ PLA University of Science and Technology \\ Nanjing, Jiangsu, 210007, China \\ ${ }^{3}$ EABAR CO., LTD \\ Weihai, Shandong, 264200, China

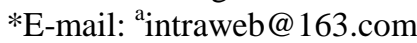 \\ E-mail: b498067717@qq.com \\ E-mail: ${ }^{c} 15266114900 @ 163 . c o m$ \\ $+*$ Corresponding author
}

\begin{abstract}
There are many types of device interfaces in the communication anti-jamming test environment, and the control instructions vary greatly. Most of the traditional test control systems are developed only for specific equipment and interfaces of a manufacturer, and its function is single. Because the reuse rate of the system is low, the cost of updating and maintaining is high. This paper proposes and designs a testing framework for the whole network based on the multi-layer software architecture, System by based on TCP protocol and socket interface command flow, implementation of all kinds of network test equipment centralized control and status monitoring, improve the accuracy and efficiency of data transmission test system of anti-jamming in the field of communication.
\end{abstract}

Keywords-multitier architecture; anti-jamming communication; integrated test

\section{INTRODUCTION}

\section{A. Communication Equipment Network Test System}

At present, network technology represented by TCP/IP, with its mature, open and other advantages, is being applied in automation in various fields, to replace the traditional mainstream serial communication automation system communication [1]. In order to ensure the normal operation of communication equipment, emerge as the times require monitoring system of communication equipment, but with the communication equipment, instrument automation, large-scale, high speed and complexity, as well as the communication network scale is more and more big [2], connected to the communication network in the communication equipment of various kinds and number of rapid growth, which makes the structure of the communications network is more complex, state monitoring communication equipment becomes very complex.

In recent years, with the development of automatic testing and integration testing of communication anti jamming techniques, in anti-jamming communication integration testing type communication equipment, instrument relates to many kinds of type, variety, the equipment monitoring and testing system has put forward higher requirements. But the equipment monitoring and testing system previously developed mostly for single or single class network equipment, scalability, flexibility [3], generality, and between each equipment monitoring and testing system are independent of each other, the data format and communication format is not the same, the "information island" effect greatly limits the equipment monitoring and testing the scope of application system anti-jamming communication, and caused a huge waste of resources.

\section{B. Multitier Architecture}

In software engineering, multitier architecture (often referred to as n-tier architecture) is a client-server architecture in which presentation, application processing, and data management functions are physically separated. The most widespread use of multitier architecture is the three-tier architecture.

$\mathrm{N}$-tier application architecture provides a model by which developers can create flexible and reusable applications. By segregating an application into tiers, developers acquire the option of modifying or adding a specific layer, instead of reworking the entire application. A three-tier architecture is typically composed of a presentation tier, a domain logic tier, and a data storage tier.

While the concepts of layer and tier are often used interchangeably, one fairly common point of view is that there is indeed a difference. This view holds that a layer is a logical structuring mechanism for the elements that make up the software solution, while a tier is a physical structuring mechanism for the system infrastructure [4][5].

In this paper, a visual communication anti-jamming device integration test system based on network environment is designed and integrated with the characteristics of the service of Communication Anti-jamming test system. Integrated test system anti-jamming equipment of the communication is designed through the design of integrated all kinds of equipments and instruments in the implementation of interface adapter, a 
plurality of communication devices, instrument communication network parameter configuration, unified management, overall monitoring and integration testing, testing management of the complex variable and simple but more effective.

\section{ANTI JAMMING SYSTEM ARCHITECTURE}

The system in this paper realizes Communication Anti-jamming infield test. Anti jamming capability of communications equipment in field test is divided into basic technical parameter test and ability of anti disturbance simulation performance tests. The basic technical indicators testing is mainly refers to the use of various instruments on the sensitivity, selectivity, double signal frequency rejection ratio, mirror frequency suppression ratio, blocking, reciprocal mixing, the average transmission power [6], frequency hopping rate were measured; the anti-interference ability simulation performance tests are tested in the simulation of interference environment loading under the communication efficiency the communication equipment, instrument, including data communication frame loss, error test, speech communication vocabulary definition, sentence intelligibility test.

The system is divided into interface layer, testing process monitor layer, data layer and intelligent equipment control layer, interface layer, the testing process monitor layer needs to be able to obtain real-time data intelligent device control layer, and the analysis of control strategy, control

commands through communication equipment

network to intelligent equipment control layer, control of intelligent equipment implementation, this is the monitoring system data exchange function. From the longitudinal data interaction, including the interface layer and control layer between the test processes, test the communication between process control layer and the intelligent equipment control layer; from the horizontal data interaction, communication test process monitoring layer internal [7]. Fig. 1 for anti jamming system architecture diagram.

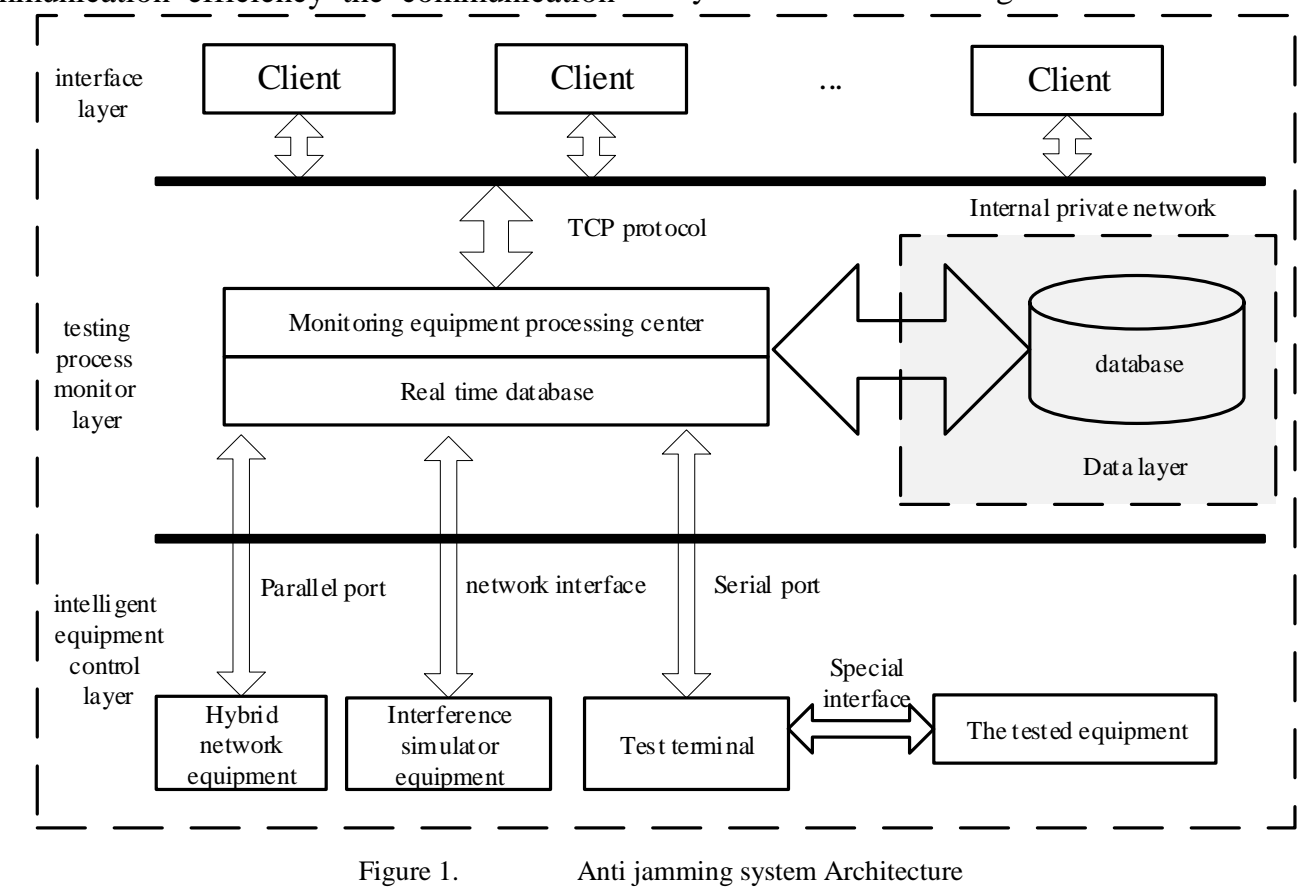

The working flow of the system: the client monitor equipment by certain time-frequency, equipment monitoring center receives the query or command, it will be a combination of query or command and control sequences, thereby forming device control instruction stream, and then follow each device control protocol instruction stream send to the end of each device via Ethernet. Through the use of instruction serialization, we can greatly reduce the difficulty of unified control different devices.

\section{REALIZATION OF NETWORK COMMUNICATION BASED ON SERIALIZATION PROCESS}

In this paper, the Anti jamming system uses the tcp protocol to build a basic network communication to ensure accurate data transmission. Specific programming communication is achieved by the socket. To accomplish this goal, we use a technique called as object serialization. For example, in the communication device simulation and control system, various exchanges between systems inside the device control information, parameter information, status information and data information, after using serialization technology, information is becoming binary serialized bytes sent over the network to facilitate [8].

Serialization steps flow instructions in simulation test system is divided into three parts: Project serialization, equipment instructions and instruction serialization, as shown in Fig. 2. Since an automation communication equipment testing will be in accordance with the timing has to perform multiple test projects or tasks [9], each test projects or tasks included in the test equipment is not the same, and each equipment in different test scenarios need to execute instructions or configuration parameters are not the same, Fig. 2 gives a typical instruction stream process to serialize. For example, communication efficiency test contains five test equipment, which are arranged in order to start, which executes instructions test automation in the terminal equipment in standby, parameter setting, start, obtain and termination of these five forms of instruction, 
through take this multi-level serialized form [10], can be every instruction in the testing process of serialization description and storage, so as to realize the rapid implementation of automation simulation and testing.

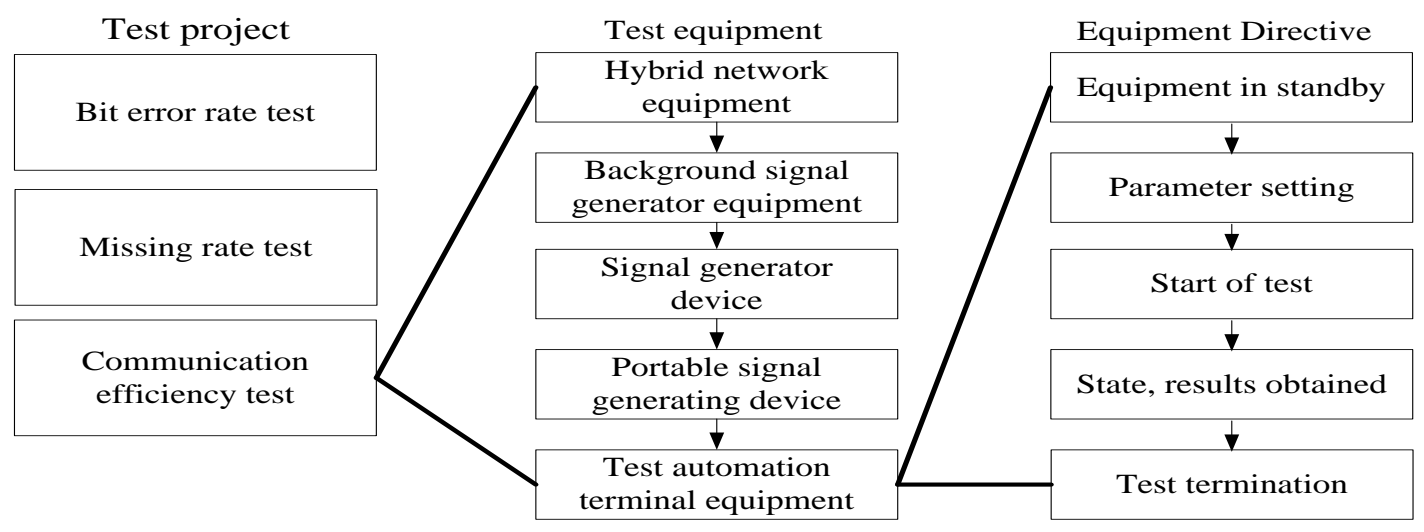

Figure 2. The serialization process instruction stream

After serialize the monitoring equipment instructions, the byte code of parameter configuration instructions will be stored into database, by this way the user can set a good test content, test environment configuration, etc. information is stored, to facilitate the bulk, mass communications test [11]. During the test, we just read from the database in the data stream forms of instruction out of the frame, converting a simple byte memory space that can continuously send data directly to the network.

In anti jamming system, internal equipment exchange of various monitoring information including control information, parameter information, the state information and data information, transfer to a frame of information form. Frames of information from various information items according to certain structure mode combination, called the information frame structure [12]. The information from the frame header contains information frame it and it appears in the information frame in the position to jointly determine the physical meaning, information items using some type of data to express.

Information can be utilized in a variety of data types are used to express the information. In order to reduce the pressure on the communication channel, information storage, computing equipment as far as possible, to improve system's real-time performance, information data types used in the system of unified communication protocol using a binary data format, a binary data format generated provides precondition for instruction stream. Fig. 3 shows a typical message frame structure.

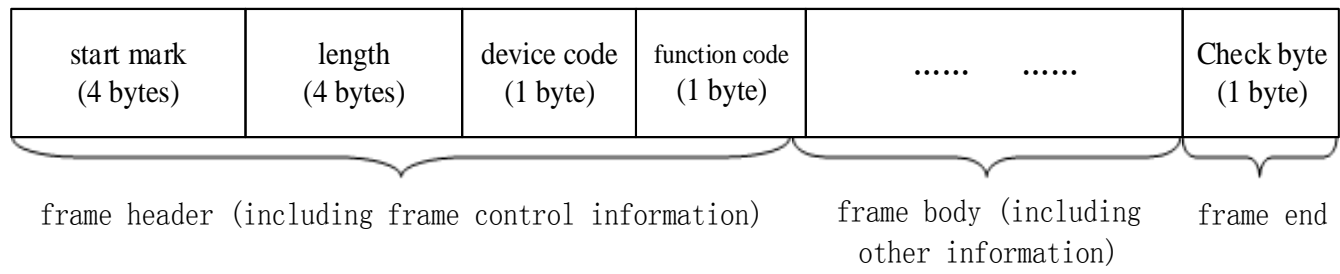

Figure 3.

Information frame structure

\section{CONCLUSION}

Anti jamming communications test and monitoring software was developed using Delphi7.0 development tools, through the use of the design of multi tier architecture, simplifying the display interface, device control and data processing software of the coupling relationship, which makes the layer code maintainability and robustness is greatly improved. At the same time, by using the method of instruction serialization, the control instructions of the device are binary and stored in the local database, which improves the efficiency of parsing, judging and processing the instructions.

With the increase of device type and communication networking mode, it is possible to test the synchronization of multi class devices in the testing process. Because the framework proposed in this paper is mainly aimed at the anti jamming test of communication in single networking mode. In the scenario where multiple devices are networked, the response time of the device will be extended, and in this case, the framework needs to be improved in conjunction with multithreading.

\section{REFERENCES}

[1] Oracle Sun Developer Network (SDN) "http://java.sun.com/developer/technicalArticles/Programming/seri alization/" (accessed on Feb 14, 2012)

[2] Axel Naumann; Philippe Canal"C++ and Data",Proceedings of Science, FERMILAB-CONF-08-692-CD

[3] Santos J R, Janakiraman G, Turner Y. Method and apparatus for enhanced design of multi-tier systems: US, US7698402[P]. 2010. 
[4] Fowler M, Rice D, Foemmel M. Patterns of Enterprise Application Architecture[J]. Pearson Schweiz Ag, 2002.

[5] Yang Fu-qing, Mei Hong and Li Ke-qing, Software Reuse and Software Component Technology. Chinese Journal of Electronics, 27(2)(1999),68-75

[6] G. Jung, K. Joshi, M. Hiltunen, R. Schlichting, and C. Pu, "A cost-sensitive adaptation engine for server consolidation of multi-tier applications," in Proc. of Middleware, 2009.

[7] Ross, J.C.; Chandran, P, "Object Serialization support for object oriented java rocessors" International Symposium on Information Technology, 2008. ITSim 2008.

[8] Opyrchal, L.; Prakash, A.; ,"Effective Object Serialization in Java", 19th IEEE International Conference on Distributed Computing Systems Workshops on Electronic Commerce and Web-based Applications/Middleware, 1999.

[9] Java ${ }^{\mathrm{TM} O b j e c t S e r i a l i z a t i o n}$ Specification, Revision 1.4.4, http://java.sun.com/j2se/1.4/pdf/serial-spec.pdf. (Accessed on Feb 14, 2012)

[10] Y. Lu, D. H.-C. Du, C. Liu, and X. Zhang, "Qos scheduling for networked storage system," in Proc. of ICDCS, 2008

[11] Y. Lu, T. F. Abdelzaher, and A. Saxena, "Design, implementation, and evaluation of differentiated caching services," IEEE Trans. on Parallel and Distributed Systems, vol. 15, pp. 440- 452, 2004.

[12] Kazuaki Maeda; " Comparative Survey of Object Serialization Techniques and the Programming Supports", World Academy of Science, Engineering and Technology 602011 